\title{
New generation of photosensitizers: the conjugate of folic acid with nanoparticles and chlorine E6
}

\begin{abstract}
Synthesis of chlorine e6 conjugates with diamond nanoparticles and folic acid. was developed. In the result of the conducted experimental investigations it was shown that chlorine e6 in a composition of chlorine e6-diamond nanoparticles-folic acid nanocomplex posess photodynamic activity, conjugated Che6 is capable of effective photosensitizing of necrotic death of tumor Hela cells in vitro at relatively low concentration and folic acid provides binding of the nanocomplex with cells expressing the folate receptor.
\end{abstract}

Keywords: nanodiamonds, nanoparticles, nanocomplex, generation drugs, conjugation

\author{
Volume 4 Issue I - 2017 \\ Lapina VA, Pavich TA, Bushuk SB, Vorobey AV, \\ Bushuk BA \\ B.I. Stepanov Institute of Physics, NASB, Belarus
}

Correspondence: Lapina VA, B.I. Stepanov Institute of Physics, NAS Belarus, Email vlapina@dragon.bas-net.by

Received: September 08, 2017 | Published: September 28 2017
Abbreviations: ND, nanodiamonds; Che6, chlorine e6; PI, propidium iodide

\section{Introduction}

Rapid development of nanotechnologies in recent years has offered a number of challenges concerning the elaboration of new generation drugs, particularly, photodynamically active ones. The use of nanoparticles offers various possibilities for the design of new generation drugs owing to reasonable combination of physical, chemical (such as adsorption, chemistry of surface, activity, safety and so on) and biological properties of components compiling a drug. In order to increase general PDT efficiency, photosensitizers are conjugated to nanoparticles of various natures (liposomes, dendrimers, fullerenes, quantum dots and others). Among new generation nanomaterials used for design of drugs Nanodiamonds (ND) of denotation synthesis occupy a special place. Earlier we have shown that the conjugation of well-known and clinically approved chlorine e6 (Che6) photosensitizer with diamond nanoparticles leads to the formation of efficient photodynamically active conjugate. ${ }^{1}$ At the same time it was established ${ }^{2}$ that ND particles conjugated with folic acid are efficiently bound to Hela cells and are able to penetrate the cells. Taking into account the above-mentioned approach of Che6ND utilization to increase PDT efficiency it is reasonable to develop a selective conjugate containing also a vector ligand, particularly, FA. The purpose of the present investigation is to determine the potential possibility of the development of new generation selective photosensitizers based on three-component Che6-ND-FA nanocomplex.

The investigation of binding of the Che6-ND-FA conjugate with cells was conducted using the methods of fluorescence microscopy and laser scanning confocal microscopy. The data obtained using confocal microscopy (Figure 1) testifies the accumulation of the nanocomplex in cells. The cells under excitation at $488 \mathrm{~nm}$ also exhibit weak autofluorescence. Upon addition of the nanocomplex to the celullar medium the increase of intracellular fluorescence is observed. These data confirm the binding of the conjugate by cells.

The fluorecence spectra of separate cells as registered using confocal microscope, coincides with that of Che 6 with maximum at $663 \mathrm{~nm}$ and shoulder at $700 \mathrm{~nm}$. The data obtained testify that
Che6-ND-FA conjugate interacts with Hela cells. The mechanism of this interaction is of considerable scientific and practical interest in view of PDT applications using synthesized conjugate. The above presented data testifies that folic acid provides binding of the conjugate with cells and its delivery into the cells and allows one to suppose the possibility of photosensitized damage of cells bound with the conjugate at their illumination. To check this assumption the conjugate photosensitized cell death was investigated. The malignant transformed Hela cells expressing the folate receptor were used. The cells were cultivated in 199 medium and were used at the logarithmic stage of their growth. For the analysis of photosensitized death of cells, $0.02 \mathrm{ml}$ of suspension of Che6-ND-FA nanocomplex ( 0.14 mass $\%$ of nancomplex containing $2 \cdot 10^{-6} \mathrm{~mole} / 1$ of Che6) was added to $0.2 \mathrm{ml}$ of cell suspension. The samples were incubated for 20 minutes to allow binding of the nanocomplex with cells. The illumination of samples was performed in HUNK camera at the thickness of optical layer of $1 \mathrm{~mm}$ by the light of KGM lamp $(150 \mathrm{~W})$ through water filter and band pass optical filter transmitting light $370-430 \mathrm{~nm}$ spectral region. The power density of incident radiation within the chlorine absorption region of Soret band was $10 \mathrm{~mW} / \mathrm{cm}^{2}$.

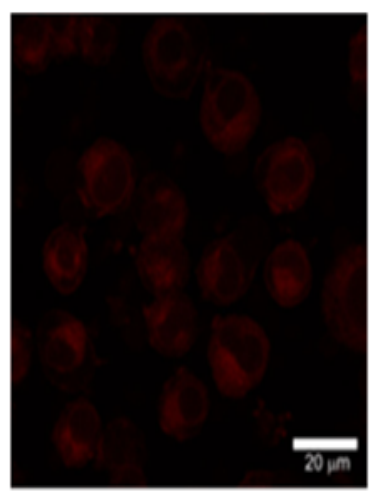

(A)

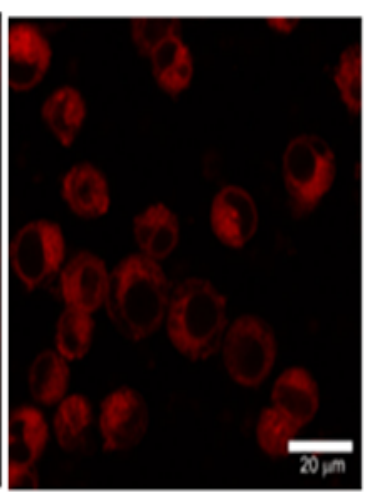

(B)
Figure I: Laser scanning microscopy of Hela cells.

A. Immediately after introduction of Che6-ND-FA nanocomplex into cell medium and

B. After incubation with the nanocomplex in darkness for 20 min. Excitation wavelength is $488 \mathrm{~nm}$. Che6 concentration is $2 \cdot 10^{-7} \mathrm{~mole} / \mathrm{l}$, nanocomplex concentration is 0.014 mass $\%$. 
The death of cells was determined by their staining with propidium iodide (PI), which was performed as follows. Previously illuminated cells were incubated for 20 minutes in darkness and then $2 \cdot 10^{-5} \mathrm{~mole} / 1$ of PI was added into the incubation medium. In 30 minutes the cells were imaged using fluorescence microscope at the wavelength region of PI fluorescence. PI was exited by green light and its fluorescense was registered in red region. PI staining was be used to quantitate cell death. The quantities of death cells (in \%) were estimated by cell counting with BD FACS Aria II flow cytometry (BD Biosciences, San Jose, USA).

Figure 2 shows the images of Hela cells suspension illuminated in the presence of Che6-ND-FA conjugate and stained by PI. As can be seen from the figure the illuminated sample exhibits the effect of nanocomplex photosensitizing action since PI cell fluorescence is registered. The presence of PI fluorescence confirms the damage of cell membranes that allows PI to enter into the cells and bind with nucleic acid with the formation of fluorescent complex. The percentage of dead cells depends on the efficiency of photodynamic action. In Table 1 the data are given on the dependence of the percentage of dead cells on sample illumination time.

Table I The dependence of Che6-ND-FA bound Hela cells death percentage on illumination time

\begin{tabular}{ll}
\hline Illumination time, Min. & \% of Dead cells \\
\hline 0 & $8.2 \pm 1.1$ \\
2 & $15.1 \pm 2.3$ \\
4 & $28.6 \pm 4.6$ \\
6 & $45.3 \pm 8.4$ \\
8 & $69.1 \pm 6.0$ \\
10 & $91,3 \pm 5.8$ \\
\hline
\end{tabular}

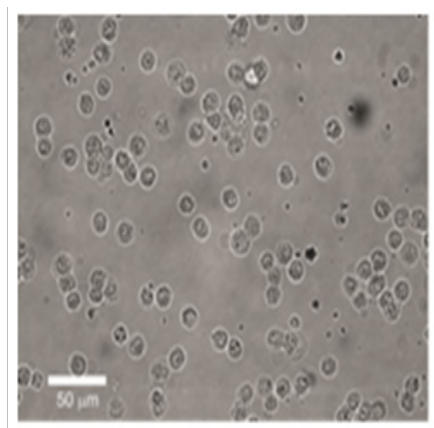

(A)

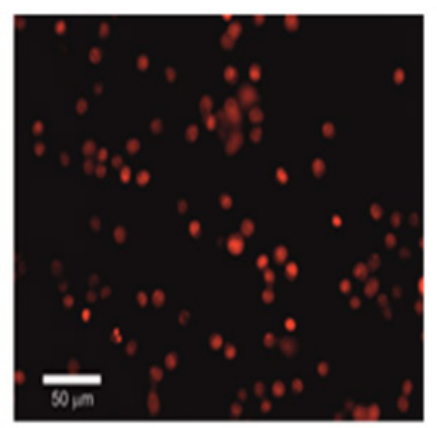

(B)
Figure 2 Microscopic images of Hela cells obtained in transmitted $(A)$ and fluorescence (B) light after Photosensitizer action of the conjugate. The time of sample illumination is 7 minutes.

\section{Conclusion}

It follows from this data that conjugated Che6 is capable effectively photosensitize the death of tumor HeLa cells in vitro at relatively low concentration $\left(2 \cdot 10^{-7} \mathrm{~mole} / \mathrm{l}\right)$.

\section{Acknowledgements}

None.

\section{Conflict of interest}

The author declares no conflict of interest.

\section{References}

1. Lapina VA, Bushuk SB, Pavich NA, et al. New generation of Photosensitizers: conjugates of chlorine e6 with diamond nanoparticles. $J$ Applied Spectroscopy. 2016;83(3):344-349.

2. Lapina VA, Vorobey AV, Pavich NA, et al. Targeting diamond nanoparticles into folate-receptor expressing Hela cells. J Applied Spectroscopy. 2013;80(3):414-418. 